Random Oper. and Stoch. Equ., Vol. 13, No. 1, pp. 17-38 (2005)

(c) VSP 2005

\title{
Subordination of symmetric quasi -regular Dirichlet forms
}

\author{
Sergio ALBEVERIO ${ }^{1,2,3,4}$ and Barbara RÜDIGER ${ }^{2,5,6}$ \\ ${ }^{1}$ Institut für Angewandte Mathematik der Universität Bonn \\ Wegelerstr. 6, D-53115 Bonn, Germany; SFB 256, SFB 611 \\ SFB 237 Essen-Bochum-Düsseldorf, Germany \\ ${ }^{2}$ BiBoS-Research Centre, D-33615 Bielefeld, Germany \\ ${ }^{3}$ CERFIM, Locarno, Switzerland \\ Acc. Arch., USI, Switzerland \\ ${ }^{4}$ Istituto di Matematica, Universitá di Trento , Italia \\ ${ }^{5}$ Mathematisches Institut, Universität Koblenz-Landau, Campus Koblenz \\ Universitätsstrasse 1, 56070 Koblenz, Germany \\ ${ }^{6}$ SFB 611, Institut für Angewandte Mathematik der Universität Bonn \\ Wegelerstr. 6, D-53115 Bonn, Germany
}

Received for ROSE October 6, 2004

\begin{abstract}
The generators of subordinate symmetric (sub-) Markov processes and their domains are exhibited by using spectral theory. The construction preserves sets of essential self -adjointness of the generators.

General non local symmetric quasi regular Dirichlet forms and the corresponding processes (with jumps) are shown to be constructible by subordination of processes properly associated to symmetric quasi regular Dirichlet forms (in particular local ones). It is proven that subordination preserves the property of a process to be a symmetric $m$-tight special standard process. A characterization of the subordinate processes in terms of solutions of the corresponding martingale problems is obtained.
\end{abstract}

Keywords: Infinite dimensional, stochastic differential equations, stochastic partial differential equations, subordination, pseudo -differential operators, Dirichlet forms, uniqueness

\section{INTRODUCTION}

The theory of (symmetric quasi -regular) Dirichlet forms provides a unified framework for constructing and discussing "nice" processes on finite and infinite dimensional state spaces, even in the case where the associated generators have non regular coefficients. The state space can be an arbitrary Hausdorff topological space equipped with a $\sigma$ -finite, positive measure. The quasi -regularity property is the analytic counterpart for having càdlàg strong Markov processes (with additional properties, technically making up what are called " $m$-tight special, standard processes", see e.g. [37], [1] and references therein). The processes are solutions of corresponding martingale problems, and in this sense the theory extends to singular coefficients, resp. infinite dimensional state spaces 
the well known relations between Feller semigroups, Hunt processes and martingale problems, see [4], [5],[6], [7], [9], [16].

Detailed properties, especially with regards to applications, of the processes and generators involved have up to now been worked out especially well for the case of local Dirichlet forms, i.e. for the case of diffusion processes. In many problems however, in mathematical physics as well as, e.g. , in biology and mathematical finance theory, processes with jumps play an increasing rule, see e.g. [21],[22],[47], [50]. The theory of Dirichlet forms provides the possibility of constructing processes with jumps (see e.g. [11]), associated with non local Dirichlet forms (by Beurling -Deny formula, see e.g. [26], [37], any quasi -regular (q.r.) Dirichlet form on a "manifold like" space splits into a local and non local part). However the construction in this way of processes with jumps in the infinite dimensional case or the finite dimensional case with singularities has not been yet pursued with the same intensity as in the case of diffusions. In the present paper we provide a systematic way to construct such examples by subordination, starting from known cases (e.g. diffusions). More precisely, we study subordination of a given quasi -regular symmetric Dirichlet form in the general setting and show that in this way one again obtains a large class of quasi -regular symmetric Dirichlet forms, with known generators and other properties which can be deduced from those of the original Dirichlet form. The technique of subordination was introduced by Bochner in 1952 and applied first in the case of locally compact spaces to construct a (subordinate) sub -Markov semigroup "associated" to a process with jumps, by changing the time (through a "subordinator") of a starting sub -Markov semigroup (see also [14], [17], [18], [20], [25], [28], [29], [31], [36], [45], [46] and references there). Here we prove that subordination preserves the property of a process to be a symmetric m-tight special standard process. In particular non -local q.r. DFs are obtained by subordinating symmetric diffusion processes, i.e. local Dirichlet forms. We characterize the subordinate processes through the corresponding martingale problems, on any set of essential self -adjointness for the generator of the starting process. Our results hold in infinitely dimensional spaces but yield also new applications for the case where the state space is finite dimensional (especially concerning the domain of the generator of the subordinate process). Some of the results of the present paper were announced in [9] or [44]. In [8] we apply our general results for the concrete construction of SDEs with non Gaussian white noise (and the corresponding unique pathwise solutions) obtained by subordination of (generalized) Ornstein -Uhlenbeck processes.

\section{SUBORDINATION OF SUB-MARKOV SEMIGROUPS}

This Section recalls the basic concepts of the theory of subordination of (sub-Markov semigroups), see e.g. [14], [31], [45] and references therein for more details.

Definition 2.1 A subordinator is a vaguely continuous convolution semigroup of probability measures $\left(\mu_{t}^{f}\right)_{t \geq 0}$ on $[0, \infty)$, with $\mu_{0}=\delta_{0}$, such that the Laplace transform $\tilde{\mu}_{t}^{f}$ is given in terms of a Bernstein function $f$, through :

$$
\tilde{\mu}_{t}^{f}(x):=\int_{0}^{\infty} \exp (-s x) \mu_{t}^{f}(d s)=\exp (-t f(x))
$$

A Bernstein function $f$ is (uniquely) represented in the following way:

$$
f(x)=b x+\int_{0}^{\infty}(1-\exp (-x s)) \quad \mu^{f}(d s)+c
$$


with $b, c \geq 0$, and $\mu^{f}$ a non negative Borel measure on $(0, \infty)$, which satisfies

$$
\int_{0}^{\infty} \frac{s}{(1+s)} \mu^{f}(d s)<\infty
$$

For the following statement and Definition see, e.g., [45], [32](p. 172ff):

Definition 2.2 A stochastic process $(y(t))_{t \geq 0}$ (with state space $\mathbb{R}$ ) on a probability space $(\Omega, \mathcal{F}, P)$ is a Lévy process in law, if it is stochastically continuous, has independent increments, $y(0)=0$ a.s., the distribution of the increments $y(t+s)-y(t)$ does not depend on $s$. $(y(t))_{t \geq 0}$ is a Lévy process if it is a Lévy process in law, and there is a set $L \in \mathcal{F}$ with $P(L)=1$, s.t. $y(t)(\omega)$ is càdlàg (i.e. is right continuous in $t \geq 0$ and has left limits in $t>0, \forall \omega \in L)$.

Theorem 2.3 There is a one to one correspondence between increasing (as a function of time) Lévy processes $(y(t))_{t \geq 0}$ on $\mathbb{R}_{+}$and Bernstein functions: the distribution $\mu_{t}^{f}$ of an increasing Lévy process $y(t)$ on $\mathbb{R}_{+}$has Laplace transform (1) given in terms of a Bernstein function $f$, and vice versa any $\left(\mu_{t}^{f}\right)_{t \geq 0}$, whose Laplace transform is given in terms of a Bernstein function through (1), is the distribution of an increasing Lévy process on $\mathbb{R}_{+}$.

Remark 2.4 From Bochner's Theorem, applied to the Fourier transform of $\left(\mu_{t}^{f}\right)_{t \geq 0}$, it follows that the finite -dimensional distributions of $(y(t))_{t \geq 0}$ define a unique measure $\nu^{f}$ on the Skorohod space $D\left[\mathbb{R}_{+} \rightarrow \mathbb{R}\right]$, so that there is in particular a canonical process with the above properties, i.e. a canonical Lévy process associated to $\left(\mu_{t}^{f}\right)_{t \geq 0}$.

Let $\left(T_{t}\right)_{t \geq 0}$ be a symmetric sub -Markov semigroup on $L^{2}(E, m) \equiv L^{2}(E, \mathcal{B}, m)$, with $(E, \mathcal{B}, m)$ any measure space. When $E$ is a topological vector space we take $\mathcal{B}=\mathcal{B}(E)$, the corresponding Borel $\sigma$-algebra. $(\cdot, \cdot)_{L^{2}(E, m)}$ (resp. $\|\cdot\|_{L^{2}(E, m)}$ ) will denote the scalar product (resp. norm) in $L^{2}(E, m)$, whenever there is no danger of confusion the notation will be simplified to $(\cdot, \cdot)$ (resp.\|.\|).

Definition 2.5 (Subordinate semigroup) Let $f$ be a Bernstein function and $\left(\mu_{t}^{f}\right)_{t \geq 0}$ the corresponding convolution semigroup. Then the semigroup $\left(T_{t}^{f}\right)_{t \geq 0}$ defined by the Bochner integral

$$
T_{t}^{f} u=\int_{0}^{\infty} T_{s} u \mu_{t}^{f}(d s) \quad u \in L^{2}(E, m), t \in \mathbb{R}_{+}
$$

is called the subordinate semigroup of $\left(T_{t}\right)_{t \geq 0}$ with respect to $f$. It is a symmetric sub -Markov semigroup on $L^{2}(E, m)([42],[13],[51])$.

Remark 2.6 Given a sub -Markov semigroup of kernels on a Polish space $E$ there is a sub -Markov process associated to it and viceversa (see e.g. [12]).

Theorem 2.7 Let a (sub-Markov) process $M=\left(\Omega, \mathcal{F}_{\infty},\left(\mathbf{X}_{t}\right)_{t \geq 0},\left(P_{x}\right)_{x \in X}\right)$ be associated with the sub-Markov semigroup $\left(T_{t}\right)_{t \geq 0}$ Let $(y(t))_{t \geq 0}$ be a Lévy process independent of $\left(X_{t}\right)_{t \geq 0}$ and associated to a subordinator with Bernstein function $f$. If the process $M^{f}=\left(\Omega, \mathcal{F}_{\infty}^{f},\left(\mathbf{X}_{t}^{f}\right)_{t \geq 0},\left(P_{x}^{f}\right)_{x \in X}\right)$ is associated with the subordinate semigroup $\left(T_{t}^{f}\right)_{t \geq 0}$, then $X_{t}^{f}$ has the same finite-dimensional distributions as $X_{y(t)}$ (with $\left.X_{y(t)}(\omega) \equiv \bar{X}_{y(t)(\omega)}(\omega)\right)$. 
Proof. It is enough to prove

$$
\begin{array}{r}
E_{x}^{f}\left[e^{i \alpha_{1} X_{t_{1}}^{f}} \ldots e^{i \alpha_{n} X_{t_{n}}^{f}}\right]=E_{x}\left[e^{i \alpha_{1} X_{y\left(t_{1}\right)}} \ldots e^{i \alpha_{n} X_{y\left(t_{n}\right)}}\right] \\
\forall t_{1} \leq t_{2} \leq \ldots \leq t_{n}, t_{i} \in \mathbb{R}_{+}, i=1, \ldots, n, \alpha_{1}, \ldots, \alpha_{n} \in \mathbb{R}, n \in \mathbb{N}
\end{array}
$$

where $E_{x}\left(\right.$ resp. $\left.E_{x}^{f}\right)$ denotes the expectation w.r.t. $P_{x}\left(\right.$ resp. $\left.P_{x}^{f}\right)$ (since the Fourier transforms of finite dimensional distributions determine the whole distributions of $\left.\left(X_{t}^{f}\right)_{t \geq 0}\right)$ ). We first remark that, with $F \in C_{b}(E)$

$$
\begin{gathered}
T_{t}^{f} F(x)=\int_{0}^{\infty} T_{s} F(x) \mu_{t}^{f}(d s) \\
=\int_{0}^{\infty} E_{x}\left[F\left(X_{s}\right) / y(t)=s\right] \mu_{t}^{f}(d s) \\
=E_{x}\left[F\left(X_{y(t)}\right)\right]
\end{gathered}
$$

hence (5) holds for the case $n=1$.

We now verify that if equation (5) holds for $n$ then it holds for $n+1$, so that the statement in Theorem 2.7 follows by induction. Indeed one has

$$
\begin{aligned}
& E_{x}^{f}\left[e^{i \alpha_{1} X_{t_{1}}^{f}} \ldots e^{i \alpha_{n+1} X_{t_{n+1}}^{f}}\right] \\
& =E_{x}^{f}\left[e ^ { \sum ^ { j = 1 } \alpha _ { j } X _ { t _ { 1 } } ^ { f } } E _ { X _ { t _ { 1 } } ^ { f } } \left[e^{\left.\left.i \alpha_{2} X_{y\left(t_{2}-t_{1}\right)} \ldots e^{i \alpha_{n+1} X_{y\left(t_{n+1}-t_{1}\right)}}\right]\right]}\right.\right. \\
& =T_{t}^{f}\left[e^{\sum_{j=1}^{n} \alpha_{j} x} E_{x}\left[e^{\left.i \alpha_{2} X_{y\left(t_{2}-t_{1}\right)} \ldots e^{i \alpha_{n+1} X_{y\left(t_{n+1}-t_{1}\right)}}\right]}\right]\right.
\end{aligned}
$$

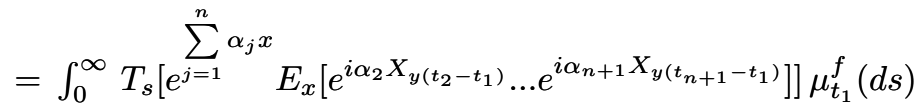

$$
\begin{aligned}
& =\int_{0}^{\infty} E_{x}\left[e^{\sum_{j=1}^{n} \alpha_{j} X_{s}} E_{X_{s}}\left[e^{i \alpha_{2} X_{y\left(t_{2}-t_{1}\right)}} \ldots e^{i \alpha_{n+1} X_{y\left(t_{n+1}-t_{1}\right)}}\right]\right] \mu_{t_{1}}^{f}(d s) \\
& =\int_{0}^{\infty} E_{x}\left[e^{\sum_{j=1}^{n} \alpha_{j} X_{s}} E_{X_{s}}\left[e^{i \alpha_{2} X_{y\left(t_{2}-t_{1}\right)}} \ldots e^{i \alpha_{n+1} X_{y\left(t_{n+1}-t_{1}\right)}}\right] / y\left(t_{1}\right)=s\right] \mu_{t_{1}}^{f}(d s) \\
& =E_{x}\left[e^{\sum_{j=1}^{n} \alpha_{j} X_{y\left(t_{1}\right)}} E_{X_{y\left(t_{1}\right)}}\left[e^{i \alpha_{2} X_{y\left(t_{2}-t_{1}\right)}} \ldots e^{i \alpha_{n+1} X_{y\left(t_{n+1}-t_{1}\right)}}\right]\right] \\
& =E_{x}\left[e^{i \alpha_{1} X_{y\left(t_{1}\right)}} \ldots e^{i \alpha_{n+1} X_{y\left(t_{n+1}\right)}}\right]
\end{aligned}
$$

(where in (9) we have used that the distribution of $X_{t_{i+1}}^{f}-X_{t_{i}}^{f}$ is the same as the one of $X_{t_{i+1}-t_{i}}^{f}$, in (13) we have used that the process is independent of the process $X$, in (9) we have used the induction hypothesis, and in (14) we have used (6)-(8)).The theorem is proved.

Remark 2.8 From Kolmogorov's Theorem (see e.g. [12]) it follows that in case $E$ is a Polish space, $\left(X_{y(t)}\right)_{t \geq 0}$ defines a unique probability measure $P^{f}$ on $\left(E^{\mathbb{R}_{+}}, \mathcal{B}\left(E^{\mathbb{R}_{+}}\right)\right)$, and a process $\left(\omega_{t}^{f}\right)_{t \geq 0}$ with state space $E$ on $\left(E^{\mathbb{R}^{+}}, \mathcal{B}\left(E^{\mathbb{R}^{+}}\right), P^{f}\right)$, such that $\omega_{t}^{f}(\omega)=$ $\omega(t), \forall \omega \in E^{\mathbb{R}^{+}} . \quad\left(\omega_{t}^{f}\right)_{t \geq 0}$ is the "canonical process corresponding to the finite dimensional distributions of $\left(X_{t}^{f}\right)_{t \geq 0}$ ". 


\section{GENERATION OF SUBORDINATE SYMMETRIC SUB-MARKOV SEMIGROUPS}

Even if we assume that $E$ is a Polish space Remarks 2.6, 2.8 do not completely inform us about the properties of the subordinate process $\left(X_{t}^{f}\right)_{t \geq 0}$, e.g., whether there is an equivalent "nice" process, and about how the corresponding generator $\left(L^{f}, \operatorname{Dom}\left(L^{f}\right)\right)$ looks like. Also it should be clarified whether the process uniquely satisfies a martingale problem for $\left(L^{f}, D\right)$ on a "good" subset $D \subset \operatorname{Dom}\left(L^{f}\right)$, how this martingale looks like, e.g., whether it is driven by a non Gaussian white noise.

In the case where $E$ is locally compact we could however try to answer part of the above questions by classical methods. In fact for the construction of Hunt processes on locally compact spaces through the semigroup theory one usually proceeds by, e.g., proving the Feller property (and then use correspondence between Feller semigroups, Hunt processes and (classical) martingale problems (MPs) on such spaces). In the case where the state space is not locally compact, it is however not clear a priori in which form this correspondence holds. Below we shall provide answers to these questions. We recall that a contraction semigroup is symmetric iff its generator is positive and self-adjoint. Below we shall prove the following:

Theorem 3.1 Let $L$ denote the generator of a symmetric sub-Markov semigroup $\left.\left(T_{t}\right)_{t \geq 0}\right)$ on $L^{2}(E, m)$. Let $f$ be a Bernstein function and let Dom $\left(L^{f}\right)$ be the generator of the subordinate sub-Markov semigroup $\left(T^{f}\right)_{t \geq 0}$ then

$$
\left(L^{f}, \operatorname{Dom}\left(L^{f}\right)\right)=(-f(-L), \operatorname{Dom}(f(-L)))
$$

where

$$
-f(-L)=-\int_{0}^{\infty} f(\lambda) d P_{\lambda},
$$

and $P_{\lambda}$ is the projection valued measure associated to the operator $-L$.

Remark 3.2 Characterizations of domains of generators $L^{f}$ of (non necessarily symmetric) subordinate (sub -Markov) semigroups have been given before (see e.g. [20], [28]). The symmetric case with $X=\mathbb{R}^{d}, f$ a complete Bernstein function, and $L$ a pseudo - differential operator with symbol satisfying certain conditions is detailed discussed in [31] (Corollary 5.1). In [46] an extension in the direction of our Theorem 3.1 was given (even for general contraction semigroups in Banach spaces), using DunfordTaylor integrals, keeping the assumption that $f$ is a complete Bernstein function (see, however, the note added in proof on p. 395 - which appeared after the results of the present paper were announced).

Proof (of the Theorem 3.1).

The proof is based on the following Prop. 3.3, 3.5 and Lemma 3.6, 3.7:

Proposition 3.3 [42]

a) For any $u \in \operatorname{Dom}(L)$

$$
L^{f} u=b L u+\int_{0}^{\infty}\left(T_{t} u-u\right) \mu^{f}(d t)
$$

b) $\left(L^{f}, \operatorname{Dom}(L)\right)$ is an operator core for $\left(L^{f}, \operatorname{Dom}\left(L^{f}\right)\right)$. 
We remark that b) follows from a) by showing $T_{t}^{f}(\operatorname{Dom}(L)) \subseteq \operatorname{Dom}(L)$, for all $t \geq 0$.

Remark 3.4 As $\left(L^{f}, \operatorname{Dom}\left(L^{f}\right)\right)$ is by Definition 2.5 self-adjoint, Proposition $\left.3.3 \mathrm{~b}\right)$ is equivalent to the statement that $\left(L^{f}, \operatorname{Dom}(L)\right)$ is essentially self-adjoint and (denoting the closure by -)

$$
\overline{\left(L^{f}, \operatorname{Dom}(L)\right)}=\left(L^{f}, \operatorname{Dom}\left(L^{f}\right)\right)
$$

\section{Proposition 3.5}

$$
\left(L^{f}, \operatorname{Dom}(L)\right)=(-f(-L), \operatorname{Dom}(L))
$$

To prove Proposition 3.5 we need the following Lemma

Lemma 3.6 The following properties hold

i) there are two constants $c \geq 0$ and $K \geq 0$ such that

$$
(f(-L) \phi, \phi) \leq c(-L \phi, \phi)+K\|\phi\|^{2} \quad \forall \phi \in \operatorname{Dom}(L)
$$

ii) there are two constants $c_{2} \geq 0$ and $K_{2} \geq 0$ such that

$$
\|-f(-L) \phi\|^{2} \leq c_{2}\|L \phi\|^{2}+K_{2}\|\phi\|^{2} \quad \forall \phi \in \operatorname{Dom}(L)
$$

iii) $\operatorname{Dom}(L) \subseteq \operatorname{Dom}(-f(-L))$

Proof. One has the representation

$$
\operatorname{Dom}(-g(-L))=\left\{\phi \in L^{2}(E, m): \int_{0}^{\infty} g^{2}(\lambda) \quad d\left(\phi, P_{\lambda} \phi\right)<\infty\right\}
$$

for $g(x)=x$, resp. $g(x)=f(x)$. Moreover we use the following remark:

to prove that a non negative measure $\mu^{f}$ on $(0, \infty)$ satisfies condition ( 3 ) (in the definition of Bernstein function) is equivalent to prove that the following two conditions hold:

$$
\int_{0}^{1} s \mu^{f}(d s)<\infty
$$

and

$$
\int_{1}^{\infty} \mu^{f}(d s)<\infty
$$

It follows then that

$$
\begin{gathered}
f(\lambda) \leq b \lambda+\int_{(0, \infty)}|1-\exp (-s \lambda)| \mu^{f}(d s) \\
\leq b \lambda+\lambda \int_{0}^{1} s \mu^{f}(d s)+2 \int_{1}^{\infty} \mu^{f}(d s) \\
\leq c \lambda+K
\end{gathered}
$$

with

$$
c=b+\int_{0}^{1} s \mu^{f}(d s), \quad K=2 \int_{1}^{\infty} \mu^{f}(d s) .
$$

Inequality (24) implies also i), as seen using the spectral theorem for $f(-L)$, resp. $-L$. 
Moreover from (24) also property ii) follows, as the spectral theorem and the properties of the projective measures $P_{\lambda}$ imply that for any Borel function $g: \mathbb{R} \rightarrow \mathbb{C}$, which is $\left(\phi, P_{\lambda} \phi\right)$-integrable,

$$
\|g(-L) \phi\|^{2}=\int_{(0, \infty)}|g(\lambda)|^{2} d\left(\phi, P_{\lambda} \phi\right)
$$

(see, e.g., [35] Chap. VI §2). iii) is then an immediate consequence of ii). The lemma is proved.

Proof of Proposition 3.5 As $\operatorname{Dom}(L)$ is a closed linear subspace of $L^{2}(E, m)$ it is sufficient to prove that

$$
\left(L^{f} \phi, \psi\right)=(-f(-L) \phi, \psi) \quad \forall \phi \in \operatorname{Dom}(L), \psi \in L^{2}(E, m)
$$

From (18), (2) it follows, for such $\phi, \psi$,

$$
\begin{gathered}
(-f(-L) \phi, \psi)=-b \int_{0}^{\infty} \lambda d\left(\phi, P_{\lambda} \psi\right) \\
+\int_{0}^{\infty} \int_{0}^{\infty}(\exp (-t \lambda)-1) \mu^{f}(d t) d\left(\phi, P_{\lambda} \psi\right) \quad \forall \phi \in \operatorname{Dom}(L)
\end{gathered}
$$

We shall prove

$$
\int_{0}^{\infty} \int_{0}^{\infty}|\exp (-t \lambda)-1| \mu^{f}(d t) d\left(\phi, P_{\lambda} \psi\right)<\infty \quad \forall \phi \in \operatorname{Dom}(L)
$$

so that we can apply Fubini's theorem to the last term in (31), obtaining then

$$
\begin{gathered}
(-f(-L) \phi, \psi)=-b(-L \phi, \psi) \\
+\int_{0}^{\infty} \int_{0}^{\infty}(\exp (-t \lambda)-1) d\left(\phi, P_{\lambda} \psi\right) \mu^{f}(d t) \quad \forall \phi \in \operatorname{Dom}(L)
\end{gathered}
$$

Proof of (32):

$$
\begin{gathered}
\int_{(0, \infty)} \int_{0}^{1}|\exp (-t \lambda)-1| \mu^{f}(d t) d\left(\phi, P_{\lambda} \psi\right) \\
\leq \int_{(0, \infty)} \lambda \int_{0}^{1} t \mu^{f}(d t) d\left(\phi, P_{\lambda} \psi\right)+2 \int_{(0, \infty)} \int_{1}^{\infty} \mu^{f}(d t) d\left(\phi, P_{\lambda} \psi\right) \\
=-(L \phi, \psi) \int_{0}^{1} t \mu^{f}(d t)+2(\phi, \psi) \int_{1}^{\infty} \mu^{f}(d t)<\infty
\end{gathered}
$$

Using the relation between generators and semigroups we obtain

$$
(-f(-L) \phi, \psi)=b(L \phi, \psi)+\int_{0}^{\infty}\left(\left(T_{t}-1\right) \phi, \psi\right) \mu^{f}(d t)
$$

We shall prove

$$
\int_{0}^{\infty}\left(\left(T_{t}-1\right) \phi, \psi\right) \mu^{f}(d t)=\left(\int_{0}^{\infty}\left(T_{t}-1\right) \phi \mu^{f}(d t), \psi\right)
$$

so that (38) becomes (29). In fact (39) is a consequence of the following inequality

$$
\int_{0}^{\infty}\left(\left(T_{t}-1\right) \phi, \psi\right) \mu^{f}(d t)<\infty
$$


which is proven as follows

$$
\begin{gathered}
\int_{(0, \infty)}\left(\left(T_{t}-1\right) \phi, \psi\right) \mu^{f}(d t) \leq\|\psi\| \int_{(0, \infty)}\left\|\left(T_{t}-1\right) \phi\right\| \mu^{f}(d t) \\
\leq\|\psi\| \int_{0}^{1}\left\|\int_{0}^{t} T_{s} L \phi d s\right\| \mu^{f}(d t)+2\|\psi\|\|\phi\| \int_{1}^{\infty} \mu^{f}(d t) \\
\leq\|\psi\| \int_{0}^{1} t\|L \phi\| \mu^{f}(d t)+2\|\psi\|\|\phi\| \int_{1}^{\infty} \mu^{f}(d t) \\
<\infty \quad \forall \phi \in \operatorname{Dom}(L), \psi \in L^{2}(E, m)
\end{gathered}
$$

where we used the contraction property of $\left(T_{t}\right)_{t \geq 0}$. Proposition 3.5 is proved.

We will use Proposition 3.5 to prove the following

Lemma $3.7(-f(-L), \operatorname{Dom}(f(-L)))$ is a closed extension of $\left(L^{f}, \operatorname{Dom}(L)\right)$

Proof. $(-f(-L), \operatorname{Dom}(f(-L)))$ is the unique closed extension of $(-f(-L), \operatorname{Dom}(L))$, due to Lemma 3.6, ii). The statement follows then from Proposition 3.5.

Theorem 3.1 is easily proved by Lemma 3.7, since there is a unique closed self- adjoint extension of $\left(L^{f}, \operatorname{Dom}(L)\right)$, which by Remark 3.4 is given by $\left(L^{f}, \operatorname{Dom}\left(L^{f}\right)\right)$. From Lemma 3.7 it follows that $(-f(-L), \operatorname{Dom}(f(-L)))$ is a closed self-adjoint extension of $\left(L^{f}, \operatorname{Dom}(L)\right)$, so that equation $(16)$ holds. Theorem 3.1 is proved.

We now prove that the property of being essentially self -adjoint of $(L, D)$, with $D \subset$ $\operatorname{Dom}(L)$, is inherited by the operator $(f(-L), D))$, if $D$ is an operator core for $(L, \operatorname{Dom}(L))$.

Theorem 3.8 [45] If $D$ is an operator core for $(L, D o m(L))$ then it is also an operator core for $(-f(-L), \operatorname{Dom}(f(-L))$.

\section{CLOSABILITY OF SUBORDINATE DIRICHLET FORMS}

We shall consider subordination of symmetric sub-Markov semigroups $\left(T_{t}\right)_{t \geq 0}$ on $L^{2}(E, m):=L^{2}(E, \mathcal{B}, m)$, with $(E, \mathcal{B}, m)$ a given measure space. From the general theory of semigroups $\left(T_{t}\right)_{t \geq 0}$ as above (see e.g. [35], [26], [27], [37]) we know that the generator $L$ of $\left(T_{t}\right)_{t \geq 0}$ is a densely defined, self- adjoint, negative-definite Dirichlet operator (see e.g. [19], [37] Chapt. I for the definition of Dirichlet operator), and viceversa, any Dirichlet operator is the generator of a symmetric sub -Markov semigroup ([19]). (To simplify notations, from now on we simply write $A$ for an operator, with the understanding that $A$ has definition domain $D(A)$. If $A$ is taken on another domain we specify it. We also make the same convention for bilinear forms.) Moreover Dirichlet operators are also in one -to -one correspondence with symmetric Dirichlet forms (DFs) $\mathcal{E}$ on $L^{2}(E, m)$, the relation being given by

$$
\begin{gathered}
\operatorname{Dom}(\mathcal{E})=\operatorname{Dom}(\sqrt{-L}) \\
\mathcal{E}(\phi, \psi)=(\sqrt{-L} \phi, \sqrt{-L} \psi) \quad \forall \phi, \psi \in \operatorname{Dom}(\sqrt{-L})
\end{gathered}
$$

$\mathcal{E}$ is by definition the Dirichlet form corresponding to the semigroup $\left(T_{t}\right)_{t \geq 0}$ generated by $L$.

Moreover the following properties are well known:

1) $\mathcal{E}(\phi, \psi)=(-L \phi, \psi) \quad \forall \phi \in \operatorname{Dom}(-L), \psi \in \operatorname{Dom}(\mathcal{E})$

2) $\operatorname{Dom}(L)$ is an operator core for $\sqrt{-L}$

3) $\quad\|\sqrt{-L} \phi\|^{2} \leq\|-L \phi\|\|\phi\| \quad \forall \phi \in \operatorname{Dom}(L)$ 
We denote by $\mathcal{E}^{f}$ the Dirichlet form corresponding to the subordinate semigroup $\left.\left(T_{t}^{f}\right)_{t \geq 0}\right)$. From Theorem 3.1 it follows that the Dirichlet form associated to $\left(T_{t}^{f}\right)_{t \geq 0}$ is given by $\mathcal{E}^{f}$ with

$$
\begin{gathered}
\operatorname{Dom}\left(\mathcal{E}^{f}\right)=\operatorname{Dom}(\sqrt{f(-L)}) \\
\mathcal{E}^{f}(\phi, \psi)=(\sqrt{f(-L)} \phi, \sqrt{f(-L)} \psi) \quad \forall \phi, \psi \in \operatorname{Dom}(\sqrt{f(-L)})
\end{gathered}
$$

$\left.1^{\prime}\right) \quad \mathcal{E}^{f}(\phi, \psi)=(f(-L) \phi, \psi) \forall \phi \in \operatorname{Dom}(f(-L)), \psi \in \operatorname{Dom}(\sqrt{f(-L)})$

Moreover the following properties hold:

2') $\operatorname{Dom}(f(-L))$ is an operator core for $\sqrt{f(-L)}$

$\left.3^{\prime}\right)\|\sqrt{f(-L)} \phi\|^{2} \leq\|f(-L) \phi\|\|\phi\| \quad \forall \phi \in \operatorname{Dom}(f(-L))$

We prove below (after Corollary 4.6) the following:

Theorem 4.1 If $D$ is an operator core for $\sqrt{-L}$ then $D$ is an operator core for $\sqrt{f(-L)}$

Remark 4.2 This theorem was announced with a short proof in [44]. In the meantime a paper by Okura appeared [41] which also contains a proof of Theorem 4.1 for locally compact separable metric spaces $E$.

Let us denote by $\|\cdot\|_{1}^{\mathcal{E}}$ the norm induced by the form $\mathcal{E}_{1} \equiv \mathcal{E}+1$, i.e.

$$
\left(\|u\|_{1}^{\mathcal{E}}\right)^{2}=\mathcal{E}(u, u)+\|u\|^{2} \quad \forall u \in \operatorname{Dom}(\mathcal{E}) .
$$

Remark 4.3 Let $D \subset \operatorname{Dom}(\mathcal{E})$ then the following three statements are equivalent

i) if $D$ is an operator core for $\sqrt{-L}$ then $D$ is an operator core for $\sqrt{f(-L)}$

ii) if $D$ is $\|\cdot\|_{1}^{\mathcal{E}}$-dense in $\operatorname{Dom}(\mathcal{E})$ it is also $\|\cdot\|_{1}^{\mathcal{E}^{f}}$ dense in $\operatorname{Dom}\left(\mathcal{E}^{f}\right)$

iii) if $(\mathcal{E}, D)$ is closable then $\left(\mathcal{E}^{f}, D\right)$ is closable.

This is easily seen from, e.g., [35].

We recall that a symmetric positive bilinear form $(\mathcal{E}, D)$ is called closable if any Cauchy sequence in the norm $\|\cdot\|_{1}^{\mathcal{E}}$ is convergent.

In other words property ii) in Theorem 4.1 gives us information about the closability of the subordinate Dirichlet form $\mathcal{E}^{f}$.

In order to prove Theorem 4.1 we need first to state and prove further results

Remark 4.4 Properties 2), 3) and 2'), 3') in this Section together with Proposition 3.3 and Lemma 3.6 easily imply the following properties

i) $\operatorname{Dom}(L) \subset \operatorname{Dom}(\sqrt{-L})$

ii) $\operatorname{Dom}(L) \subset \operatorname{Dom}(f(-L)) \subset \operatorname{Dom}(\sqrt{f(-L)})$

iii) $\overline{(\sqrt{-L}, \operatorname{Dom}(L))}=(\sqrt{-L}, \operatorname{Dom}(\sqrt{-L}))$

iv) $\overline{(f(-L), \operatorname{Dom}(L))}=(f(-L), \operatorname{Dom}(f(-L)))$

v) $\overline{(\sqrt{f(-L)}, \operatorname{Dom}(f(-L)))}=(\sqrt{f(-L)}, \operatorname{Dom}(\sqrt{f(-L)}))$ 
A further property is easily proven similarly as for Lemma 3.6., using (22)-(24) and the spectral theorem

vi) $\operatorname{Dom}(\sqrt{-L}) \subset \operatorname{Dom}(\sqrt{f(-L)})$ and there are two constants $c \geq 0, K \geq 0$, such that

$$
\|\sqrt{f(-L)} \phi\|^{2} \leq c\|\sqrt{-L} \phi\|^{2}+K\|\phi\|^{2} \quad \forall \phi \in \operatorname{Dom}(\sqrt{-L})
$$

\section{Proposition 4.5}

$$
\text { vii) } \overline{(\sqrt{f(-L)}, \operatorname{Dom}(L))}=(\sqrt{f(-L)}, \operatorname{Dom}(\sqrt{f(-L)}))
$$

Before proving this Proposition we give the following Corollary:

\section{Corollary 4.6}

$$
\text { viii) } \overline{(\sqrt{f(-L)}, \operatorname{Dom}(\sqrt{-L}))}=(\sqrt{f(-L)}, \operatorname{Dom}(\sqrt{f(-L)}))
$$

Proof (of Corollary 4.6). From properties i) and vi) it follows

$$
\operatorname{Dom}(-L) \subset \operatorname{Dom}(\sqrt{-L}) \subset \operatorname{Dom}(\sqrt{f(-L)})
$$

so that Corollary 4.6 follows from Proposition 4.5 and the fact that $(\sqrt{f(-L)}, \operatorname{Dom}(\sqrt{f(-L)}))$ is self -adjoint and therefore also closed. Corollary 4.6 is proved.

Proof (of Proposition 4.5).

Because of property v) $\operatorname{Dom} f(-L)$ is dense in $\operatorname{Dom}(\sqrt{f(-L)})$.

By property 2') and an $\frac{\varepsilon}{2}$-argument, we then have Prop. 4.5.

\section{Proof of Theorem 4.1}

From Corollary 4.6, Dom $\sqrt{f(-L)}$ is dense in $\operatorname{Dom}(\sqrt{-L})$.

By the hypothesis that $D$ is an operator core for $\sqrt{-L}, D$ is dense in $\operatorname{Dom}(\sqrt{-L})$. An $\frac{\varepsilon}{2}$-argument then yields Theorem 4.1 .

\subsection{Subordinate quasi -regular (regular) Dirichlet forms}

From now on we assume that $E$ is a Hausdorff topological space, $\mathcal{B}=\mathcal{B}(E)=\sigma(C(E))$, with $\mathcal{B}(E)$ the Borel $\sigma$-algebra, $C(E)$ the set of continuous functions on $E$, and $m$ a $\sigma$ - finite, positive measure on $(E, \mathcal{B}(E))$. It is known that if the Dirichlet form $\mathcal{E}$ is a "quasi -regular" Dirichlet form (q.r. D.F.) [37], the process "properly associated" to $\mathcal{E}$ (or equivalently to the corresponding sub-Markov semigroup) is an $m$-tight special standard process, and has in particular the "nice" properties of being a càdlàg process which is also quasi -left continuous and strong Markov.

We give here some more details about this relation, which are needed for the concrete construction of subordinate processes. (For a more systematic presentation of all concepts related to Dirichlet forms, and their origin in potential theory, we refer to [37]. For a shorter survey and newer applications see [1]). We do not however give here the definition of " $m$-tight special standard process". We just mention that it is a suitable substitute in the case of an infinite dimensional state space $E$ of the corresponding concept of Hunt process used for locally compact spaces. In fact, in the special case where the state space is locally compact, and the life time of the process $\mathbf{M}$ is infinite, one has 
that $\mathbf{M}$ is a Hunt process (see e.g. [15]) and the corresponding q.r. DF $\mathcal{E}$ is a regular DF. (Viceversa if $E$ is locally compact, a regular DF on $L^{2}(E, m)$ is always q.r. and the corresponding Hunt process on $E_{\Delta}=E \cup \Delta$, with $E_{\Delta}$ the one -point compactification of $E$, is an m-tight special standard process).

To introduce the concept of q.r. D.F., we need some more definitions.

Definition 4.7 (Capacity). Given an open set $A \subset E$, the capacity $C a p^{\mathcal{E}}(A)$ of $A$ with respect to the Dirichlet form $(\mathcal{E}, \operatorname{Dom}(\mathcal{E})$ is given by

$$
\begin{aligned}
& \operatorname{Cap}^{\mathcal{E}}(A)=\inf _{\phi \in \mathcal{L}_{A}^{\mathcal{E}}} \mathcal{E}_{1}(\phi, \phi) \text { if } \mathcal{L}_{A}^{\mathcal{E}} \neq \emptyset \\
& \infty \quad \text { if } \mathcal{L}_{A}^{\mathcal{E}}=\emptyset
\end{aligned}
$$

where

$$
\mathcal{L}_{A}^{\mathcal{E}}:=\{\phi \in \operatorname{Dom}(\mathcal{E}): \phi \geq 1 \quad m-\text { a.e. } \quad \text { on } A\}
$$

If $N$ is any subset of $E$ then

$$
\operatorname{Cap}^{\mathcal{E}}(N)=\inf _{N \subset A \in \mathcal{O}} \operatorname{Cap}^{\mathcal{E}}(A)
$$

where $\mathcal{O}$ is the collection of open subsets of $E$.

The sets $N$ with $\operatorname{Cap}^{\mathcal{E}}(N)=0$ are precisely the sets which are never reached by the corresponding process. Moreover if $\operatorname{Cap}^{\mathcal{E}}(N)=0$ then $m(N)=0$ (but not viceversa in general). If a property holds outside such a set one says the property holds quasi -everywhere (q.e.).

Definition $4.8(\mathcal{E}-N e s t)$. An increasing sequence $\left(F_{k}\right)_{k \in N}$ of closed subsets $F_{k} \subset E$, $F_{k} \subset F_{k+1}$ is an $\mathcal{E}$-nest if

$$
\lim _{k \rightarrow \infty} \operatorname{Cap}^{\mathcal{E}}\left(F_{k}\right)=0
$$

Definition $4.9 \mathcal{E}$-exceptional set $N$ is an $\mathcal{E}$-exceptional set if $N \subset \cap_{k \in \mathbb{N}} F_{k}^{c}$, where $F_{k}^{c}$ is the complementary set of $F_{k}$, and $\left(F_{k}\right)_{k \in \mathbb{N}}$ is an $\mathcal{E}$-nest.

Remark 4.10 $N \subset E$ is $\mathcal{E}$-exceptional iff $\operatorname{Cap}^{\mathcal{E}}(N)=0$. For this see, e.g., [26].

Definition 4.11 ( $\mathcal{E}$-quasi -continuous function) A function $\phi \in L^{2}(E, m)$ is $\mathcal{E}$-quasi -continuous if there exists an $\mathcal{E}$-nest $\left(F_{k}\right)_{k \in \mathbb{N}}$ such that the function $\left.\phi\right|_{F_{k}}$, (i.e. $\phi$ restricted to $F_{k}$ ), is continuous for any $k \in \mathbb{I N}$.

Remark 4.12 $\phi \in L^{2}(E, m)$ is $\mathcal{E}$-quasi -continuous iff $\forall \epsilon>0$ there exists an open set $G$ such that $\operatorname{Cap}^{\mathcal{E}}(G)<\epsilon$ and $\left.\phi\right|_{G^{c}}$ is continuous. For this see, e.g., [26].

Definition $4.13 \mathcal{E}$-quasi-continuous $m$-version $\tilde{\phi} \in L^{2}(E, m)$ is an $\mathcal{E}$-quasi-continuous $m$ - version of $\phi \in L^{2}(E, m)$ if $\tilde{\phi}=\phi m$-a.e. and $\tilde{\phi}$ is $\mathcal{E}$-quasi -continuous. 
Definition 4.14 (Quasi -regular Dirichlet form) A symmetric Dirichlet form $(\mathcal{E}, \operatorname{Dom}(\mathcal{E})$ on $L^{2}(E, m)$ is quasi -regular if

1) there exists an $\mathcal{E}$-nest $\left(F_{k}\right)_{k \in \mathbb{N}}$ consisting of compact sets

2) there exists a $\|\cdot\|_{1}^{\mathcal{E}}$-dense subset $D$ of $\operatorname{Dom}(\mathcal{E})$ whose elements have an $\mathcal{E}$-quasi -continuous $m$-version.

3) there exists a sequence $\left(\phi_{n}\right)_{n \in \mathbb{N}}$ with $\phi_{n} \in \operatorname{Dom}(\mathcal{E})$, having $\mathcal{E}$-quasi -continuous $m$ - versions $\tilde{\phi}_{n}$, and an $\mathcal{E}$-exceptional set $N \subset E$ such that $\left\{\tilde{\phi}_{n}, n \in \mathbb{I}\right\}$ separates the points of $E \backslash N$.

Theorem 4.15 (see [37]) Let $\mathcal{E}$ be a quasi -regular Dirichlet form on $L^{2}(E, m)$, then up to $m$-equivalence there is a unique $m$-tight special standard process

$$
\mathbf{M}=\left(\Omega, \mathcal{F}_{\infty},\left(X_{t}\right)_{t \geq 0},\left(P_{z}\right)_{z \in X_{\Delta}}\right)
$$

on the extended space $E_{\Delta}$ adapted to the natural filtration $\left(\mathcal{F}_{t}\right)_{t \geq 0}$ such that $\mathbf{M}$ is properly associated with $(\mathcal{E}$, DomE $)$.

( $\Delta$ is an isolated point and is the cemetery of the process $\mathbf{M}$. If the life time of $\mathbf{M}$ is infinite $\Delta$ is never reached by $\mathbf{M}$.)

(Two right processes $\mathbf{M}$ and $\mathbf{M}^{\prime}$ with state space $E$ and transition function $p_{t}$ (resp. $\left.p_{t}^{\prime}\right)$ are " $m$-equivalent", if there is a set $S \subset \mathcal{B}(E)$, with $m(S)=1$, s.th. $S$ is $M$ - (resp. $\left.M^{\prime}-\right)$ invariant and $p_{t} f(x)=p_{t}^{\prime} f(x) \forall f \in B_{b}(E)$ and $x \in E$. [37])

\subsection{Quasi regular subordinate Dirichlet forms}

Theorem 4.16 Let $f$ be a Bernstein function. If the symmetric Dirichlet form $\mathcal{E}$ on $L^{2}(E, m)$ with generator $L$ is quasi -regular then the symmetric Dirichlet form $\mathcal{E}^{f}$ with generator $-f(-L)$ is also quasi -regular.

Proof. The proof is an easy consequence of the following Lemma, and the subsequent remarks.

Lemma 4.17 There is a constant $C \geq 0$ such that for any subset $N \subset E$.

$$
\operatorname{Cap}^{\mathcal{E}^{f}}(N) \leq \operatorname{CCap}^{\mathcal{E}}(N)
$$

Proof. From Lemma 3.6 it follows that there is a constant $C \geq 0$ such that

$$
\mathcal{E}_{1}^{f}(\phi, \phi) \leq C \mathcal{E}_{1}(\phi, \phi) \quad \forall \phi \in \operatorname{Dom}(\mathcal{E})
$$

$\left(\right.$ where $\left.\mathcal{E}_{1}^{f} \equiv \mathcal{E}^{f}+(),\right)$.

This implies inequality (58). Lemma 4.17 is proved.

Remark 4.18 From Lemma 4.17 it follows in particular that

i) any $\mathcal{E}$-nest is also an $\mathcal{E}^{f}$-nest,

ii) any $\mathcal{E}$-exceptional set is also $\mathcal{E}^{f}$-exceptional,

iii) if $\phi \in L^{2}(X, m)$ is $\mathcal{E}$-quasi -continuous then it is also $\mathcal{E}^{f}$-quasi -continuous.

Applying Remark 4.18 we see immediately that Properties 1) and 3) in Definition 4.14 follow.

Proof (of property 2 in Definition 4.14).

By hypothesis there exists an $\|\cdot\|_{1}$-dense subset $D$ of $\operatorname{Dom}(\mathcal{E})$ whose elements have an $\mathcal{E}$-quasi -continuous version. By Remark 4.18, if $\phi \in D$ then $\phi$ has also an $\mathcal{E}^{f}$ 
-quasi -continuous version. Moreover the statement that $D$ is $\|\cdot\|_{1}$-dense in $\operatorname{Dom}(\mathcal{E})$ is equivalent to the statement that $D$ is an operator core for $\sqrt{-L}$ and by Theorem 4.1 this implies that $D$ is an operator core for $\sqrt{-f(-L)}$, i.e. $D$ is an $\|\cdot\|_{1}^{f}$-dense subset of $\operatorname{Dom}\left(\mathcal{E}^{f}\right)$ The property is proved.

\subsection{Non local DFs obtained by subordination of local DF's}

In recent years an important use has been made of DFs in the construction of (strong) local DFs and the corresponding diffusion processes (see, e.g., [1], [2], [3], [6], [5], [4], [49]). For the following definitions and results see [37].

Definition 4.19 (Diffusion process) A diffusion process is an $m$-tight special standard process which satisfies the following property:

$$
P_{z}\left(X_{t} \text { is continuous intime }\right)=1 \text { for } m \text {-a.e. } \quad z
$$

Definition 4.20 (Strong local Dirichlet form) A Dirichlet form $\mathcal{E}$ is strong local if $\forall u, v \in \operatorname{Dom}(\mathcal{E})$, such that $u$ constant on every open set $I$ which contains the support $\operatorname{supp}[v]$ of $v$, it follows $\mathcal{E}(u, v)=0$.

Definition 4.21 Local Dirichlet form A Dirichlet form $\mathcal{E}$ is local if $\mathcal{E}(u, v)=0$, for all $u, v \in \operatorname{Dom}(\mathcal{E})$ with $\operatorname{supp}[u] \cap \operatorname{supp}[v]=\emptyset$

Remark 4.22 A strong local Dirichlet form is local.

Theorem 4.23 A quasi -regular Dirichlet form on $L^{2}(E, \mathcal{B}, m)$ is strong local iff the $m$-tight special standard process properly associated to it is a diffusion process.

From the following Proposition it follows that the subordinate of a local quasi regular Dirichlet form (i.e. the Dirichlet form corresponding to the subordinate semigroup) is a non local quasi regular Dirichlet form (i.e. a quasi regular Dirichlet form which is not local). The $m$-tight special standard process $\mathbf{M}^{f}$ properly associated to it is then a process with jumps.

Proposition 4.24 Suppose that $\mathcal{E}$ is a symmetric, quasi -regular, local Dirichlet form on $L^{2}(E, m)$ corresponding to the semigroup $\left(T_{t}\right)_{t \geq 0}$, let $\phi \geq 0, \phi \in \operatorname{Dom}(\mathcal{E})$, and suppose that there is a function $\psi \geq 0, \psi \in \operatorname{Dom}(L)$, s.t. $\operatorname{supp}[\phi] \cap \operatorname{supp}[\psi]=\emptyset$ then $\mathcal{E}^{f}(\phi, \psi)=0$ implies

$$
T_{t} \psi(x)=\psi(x) \quad \text { for } \quad m-\text { a.e. } \quad x \in \operatorname{supp}[\phi]
$$

Proof. From Proposition 3.3 we know that

$$
-\mathcal{E}^{f}(\phi, \psi)=b(\phi, L \psi)+\left(\phi, \int_{0}^{\infty}\left(T_{t} \psi-\psi\right) \mu^{f}(d t)\right)
$$

and from equation (39) it follows

$$
\left.-\mathcal{E}^{f}(\phi, \psi)=b(\phi, L \psi)+\int_{0}^{\infty}\left(\phi,\left(T_{t} \psi-\psi\right)\right) \mu^{f}(d t)\right)
$$


By hypothesis however $(\phi, L \psi)=0$, so that $\mathcal{E}^{f}(\phi, \psi)=0$ implies

$$
0=\left(\phi,\left(T_{t} \psi-\psi\right)\right) \geq 0 \quad \mu^{f}-\text { a.e. }
$$

so that

$$
T_{t} \psi(x)=\psi(x)=0 \quad \text { for } \quad m \times \mu^{f} \text { a.e. } \quad x \in \operatorname{supp}[\phi]
$$

and the Proposition follows then by the strong continuity of $\left(T_{t}\right)_{t \geq 0}$. Proposition 4.24 is proved.

Remark 4.25 The subordinate Dirichlet form $\mathcal{E}^{f}$ of a non local Dirichlet form $\mathcal{E}$ is non local. This can be proven easily using (20), (47), (48) and Remark 2.2.1, vi).

\section{4. "Nice" processes characterized by the corresponding martingale prob- lems}

In this Section we first recall the decomposition theorem for additive functionals related to quasi -regular Dirichlet forms (Theorem 4.26) as well as Theorem 4.28 where the corresponding (continuous) quadratic variation is given. (Theorem 4.28 is proven in [8].) These are then used to prove Theorem 4.33, which characterizes the subordinate processes in terms of the unique solutions of the corresponding martingale problems on the sets of essentially self -adjointness of the starting generator, for which subordination is performed.

Let $\mathbf{M}=\left(\Omega, \mathcal{F}_{\infty},\left(X_{t}\right)_{t \geq 0},\left(P_{z}\right)_{z \in E_{\Delta}}\right)$ be an $m$-tight special standard process properly associated to $(\mathcal{E}, \operatorname{Dom}(\overline{\mathcal{E}}))$

Let $u \in \operatorname{Dom}(\mathcal{E})$ and

$$
A_{t}^{[u]}:=\tilde{u}\left(X_{t}\right)-\tilde{u}\left(X_{0}\right)
$$

where $\tilde{u}$ is an $\mathcal{E}$-quasi continuous version of $u \in \operatorname{Dom}(\mathcal{E})$, then $\left(A_{t}^{[u]}\right)_{t \geq 0}$ is an additive functional (AF) of $\mathbf{M}$ (see Def.2.2 Chapt. $6 \S 2$ [37]).

The following result holds (Theorem 2.5 Chapt.VI [37])

Theorem 4.26 (Decomposition Theorem) If $u \in \operatorname{Dom}(\mathcal{E})$, then there exists a unique $\left(M^{[u]}\right)_{t \geq 0} \in \mathcal{M},\left(N^{[u]}\right)_{t \geq 0} \in \mathcal{N}^{c}$, such that

$$
A_{t}^{[u]}=M_{t}^{[u]}+N_{t}^{[u]}
$$

where

$$
\stackrel{\circ}{\mathcal{M}}:=\{M \in \mathcal{M} \mid e(M)<\infty\}
$$

where with $e(A)$ we denote the energy of the $A F\left(A_{t}\right)_{t \geq 0}$, i.e.

$$
e(A):=\lim _{t \rightarrow 0} \frac{1}{2 t} E_{m}\left[A_{t}^{2}\right]
$$

and with

$$
\begin{aligned}
& \mathcal{M}:=\left\{M \mid M \text { is an } \mathrm{AF} \text { of } \mathbf{M}, E_{z}\left[M_{t}^{2}\right]<\infty,\right. \\
&\left.E_{z}\left[M_{t}\right]=0, \text { for } \mathcal{E}-\text { q.e. } z \in E \text { and all } t \geq 0\right\} \\
& \mathcal{N}^{c}:=\{N: N \text { is a continuous AF s.th. } e(N)=0, \\
&\left.E_{x}\left[\left|N_{t}\right|\right]<\infty \text { q.e. for each } t>0\right\}
\end{aligned}
$$


Remark 4.27 $M_{t}^{[u]}, N_{t}^{[u]}$ are, by definition of additive functionals of $\mathbf{M}$ [37], $\mathcal{F}_{t}$ adapted, where $\left(\mathcal{F}_{t}\right)_{t \geq 0}$ is the natural filtration of $\mathbf{M}$

- For any $M \in \mathcal{M}, M_{t}^{[u]}$ is a square integrable martingale [26], [27], [37], and the (continuous part of) the quadratic variation $\left\langle M^{[u]}\right\rangle_{t}$ is a positive continuous additive functional (PCAF).

- If $u \in \operatorname{Dom}(L)$, then $\tilde{u}\left(X_{t}\right)-\tilde{u}\left(X_{0}\right)$ is a semimartingale, and

$$
N_{t}^{[u]}=\int_{0}^{t} L u\left(X_{s}\right) d s
$$

On the other hand, $\left\langle M^{[u]}\right\rangle_{t}$ can be computed by means of the following theorem proven in [8] and announced in [9], [44] which extends [4] (from the case of classical Dirichlet forms to more general Dirichlet forms):

Theorem 4.28 Let $(\mathcal{E}, \operatorname{Dom}(\mathcal{E}))$ be a symmetric quasi-regular Dirichlet form on $L^{2}(E, m)$. Suppose that $1 \in \operatorname{Dom}(L)$, where $L$ is the generator corresponding to $\mathcal{E}$, then for any $u \in \operatorname{Dom}(L)$, such that $u^{2} \in \operatorname{Dom}(L)$

$$
<M^{[u]}>_{t}=\int_{0}^{t} \rho\left(X_{s}\right) d s
$$

where

$$
\rho(x)=L u^{2}(x)-2 u(x) L u(x)
$$

Remark 4.29 In the case of locally compact spaces $E$ this was already discussed e.g. in [23]. In [19] the relation between the "carré du champ" of Dirichlet forms and Feller semigroups is discussed. The validity of the corresponding relation for right semigroups on such state spaces $E$ is mentioned in Remark 4.3.10 of [19].

The theory of DFs provides the possibility to characterize $m$-tight special standard processes by proving the existence and uniqueness of the solution to the corresponding martingale problems. In fact if "Markov uniqueness" (see [7], [24] for this concept) holds for $(\mathcal{E}, D)$ with $D \subset \operatorname{Dom}(L) \subset \operatorname{Dom}(\mathcal{E})$ where $\mathcal{E}$ is a quasi regular Dirichlet form, then the $m$-tight special standard process properly associated to $\mathcal{E}$ uniquely solves the martingale problem for the generators $L$ on $D$ [7]. If $\mathcal{E}$ is a symmetric quasi -regular DF Markov uniqueness for $(\mathcal{E}, D)$ is garanteed in case $(L, D)$ satisfies the stronger property of being essentially self -adjoint. We reformulate the statement here only for this case, and refer to [7] for the general case where Markov uniqueness holds.

Definition 4.30 $(M P$ for $(L, D)$.) Let $\mathcal{E}$ be a symmetric quasi -regular Dirichlet form with generator $L$ on $L^{2}(E, m)$, and let $D \subset \operatorname{Dom}(L)$. An $m$-tight special standard process $M=\left(\Omega, \mathcal{F}_{\infty},\left(\mathbf{X}_{t}\right)_{t \geq 0},\left(P_{z}\right)_{z \in X_{\Delta}}\right)$ satisfies the $M P$ for $(L, D)$ under $\left(P_{z}\right)_{z \in X_{\Delta}}$ if for any $u \in D$

$$
M_{t}^{[u]}:=\tilde{u}\left(\mathbf{X}_{t}\right)-\tilde{u}\left(\mathbf{X}_{0}\right)-\int_{0}^{t} L u\left(\mathbf{X}_{s}\right) d s
$$

is an $\mathcal{F}_{t}$-Martingale under $\left(P_{z}\right)_{z \in X_{\Delta}}$ for $\mathcal{E}$-q.e. starting point $z=E_{0} \in E$, where $\mathcal{F}_{t}$ is the natural filtration of $M$, and $\tilde{u}$ is any $\mathcal{E}^{f}$-quasi continuous version of $u$. 
Theorem 4.31 [7] Let $\mathcal{E}$ be a symmetric quasi -regular Dirichlet form with generator $L$ on $L^{2}(E, m)$. Assume $(L, D)$ is essentially self -adjoint for some $D \subset \operatorname{Dom}(L)$, then up to " $m$-equivalence" there is a unique $m$-symmetric, $m$-tight special standard process $M=\left(\Omega, \mathcal{F}_{\infty},\left(\mathbf{X}_{t}\right)_{t \geq 0},\left(P_{z}\right)_{z \in E_{\Delta}}\right)$ satisfying the $M P$ for $(L, D)$.

Remark 4.32 Under the same hypothesis of Theorem 4.31 there is up to "m-equivalence" a unique $m$-symmetric, $m$-tight special standard process $M=\left(\Omega, \mathcal{F}_{\infty},\left(\mathbf{X}_{t}\right)_{t \geq 0},\left(P_{z}\right)_{z \in E_{\Delta}}\right)$ satisfying the $M P$ for $(L, D)$ under $P_{m}:=\int P_{z} d m$, i.e. s.th. for any $u \in D M_{t}^{[u]}$ is an $\mathcal{F}_{t}$-Martingale under $P_{m}$.

From Theorem 4.31, Theorem 3.8, Theorem 4.28 the following Theorem follows easily:

Theorem 4.33 Let $f$ be a Bernstein function and $\mathcal{E}$ be a symmetric quasi -regular Dirichlet form with generator $L$ on $L^{2}(E, m)$. Assume that $D \subset D o m(L)$ and $(L, D)$ essentially self -adjoint. Then up to $m$-equivalence there is a unique $m$-symmetric, $m$ -tight special standard process

$M^{f}=\left(\Omega, \mathcal{F}_{\infty}^{f},\left(X_{t}\right)_{t \geq 0}^{f},\left(P_{z}\right)_{z \in E_{\Delta}}^{f}\right)$ satisfying the following $\mathcal{E}^{f}-M P$ for $(-f(-L), D)$ : for any $u \in D$

$$
M_{t}^{[u], f}:=\tilde{u}\left(X_{t}^{f}\right)-\tilde{u}\left(X_{0}^{f}\right)+\int_{0}^{t} f(-L) u\left(X_{s}^{f}\right) d s
$$

is an $\mathcal{F}_{t}^{f}$-martingale under $\left.\left(P_{z}\right)_{z \in E_{\Delta}}^{f}\right)$ for $\mathcal{E}^{f}$-q.e. starting point $E_{0}=z \in E$, where $\mathcal{F}_{t}^{f}$ is the natural filtration of $M^{f}$, and $\tilde{u}$ is any $\mathcal{E}^{f}$-quasi continuous version of $u$. $M^{f}$ is properly associated to the subordinate sub-Markov semigroup $\left(T_{t}^{f}\right)_{t \geq 0}$ If in addition $u^{2} \in \operatorname{Dom}(f(-L))$, then the quadratic variation of $M_{t}^{[u], f}$ is

$$
<M^{[u], f}>_{t}=\int_{0}^{t}\left[-f(-L) u^{2}\left(X_{s}^{f}\right)+2 u\left(X_{s}^{f}\right) f(-L) u\left(X_{s}^{f}\right)\right] d s
$$

\subsection{Example. Subordination of Ornstein -Uhlenbeck processes on $\mathbb{R}$}

In this Section we first list some known results concerning Ornstein -Uhlenbeck (OU) processes on $\mathbb{R}$ and then analyze the corresponding subordinate processes. All the results mentioned here can be easily generalized to the case of $\mathbb{R}^{d}$. We do not do it here explicitely, since this case will appear as a special case of our treatment of infinite -dimensional state spaces in [8].

Let $E=\mathbb{R}$, and $m=\mu_{a}, a>0$, a Gaussian measure on $\mathbb{R}$ with zero mean and variance $a^{-1}$. Let

$$
\mathcal{E}_{a}(u, v)=\int \nabla u \nabla v d \mu_{a} \quad \forall u, v \in \mathcal{H}_{a}
$$

where $\mathcal{H}_{a}$ is the linear span of Hermite polynomials in $\mathbb{R}$ associated to $\mu_{a}$, i.e. the set of finite linear combinations of the scaled Hermite polynomials

$$
: x^{n}: a:=H_{n}\left(x / \sqrt{a^{-1}}\right)
$$

where

$$
\begin{gathered}
H_{0}(x):=1 \\
H_{n}(x)=\frac{(-1)^{n}}{\sqrt{n !}} \exp \left(x^{2} / 2\right) \frac{d^{n}}{d x^{n}}\left(\exp \left(-x^{2} / 2\right)\right.
\end{gathered}
$$

are the normalized Hermite polynomials. 
Let

$$
: \exp (u x)::_{a}:=\exp \left(u x-a^{-1} u^{2} / 2\right)
$$

then

$$
: \exp (u x):_{a}=\sum_{n=0}^{\infty} \frac{u^{n}}{\sqrt{n !}} a^{-n / 2}: x^{n}:_{a}
$$

Remark 4.34 The so defined functions $\left(\left(\mathbb{R}, \mu_{a}\right)\right.$-random variables) : $x^{n}:_{a}$ differ from the usually defined $n$-th Wick powers random variables (see e.g. [48]) by the factor $\frac{1}{\sqrt{n !}} a^{-n / 2}$. (82) corresponds instead exactly to the usual defined Wick exponential, (83) differs from the usual relation between Wick exponential and Wick powers by the coefficient factor $\frac{1}{\sqrt{n !}} a^{-\frac{n}{2}}$ instead of $\frac{1}{n !}$.

It is well known (see e.g. [43], [19]) that the Hermite polynomials : $x^{n}:{ }_{a}, n \in \mathbb{N}$ form an orthonormal basis (ONB) in

$$
L^{2}\left(\mu_{a}\right):=L^{2}\left(\mathbb{R}, \mu_{a}\right)
$$

which is the direct sum of the subspaces

$$
\mathcal{H}_{a}^{n}:=\left\{\alpha: x^{n}: a, \alpha \in \mathbb{R}\right\}
$$

i.e.

$$
L^{2}\left(\mathbb{R}, \mu_{a}\right)=\oplus \mathcal{H}_{a}^{n}, n \in \mathbb{N} .
$$

This decomposition is called the "chaos decomposition". Moreover the following results are well known (see e.g. [19], [6], [30], [40])

Theorem $4.35\left(\mathcal{E}_{a}, \mathcal{H}_{a}\right)$ is closable in $L^{2}\left(\mu_{a}\right)$, its closure $\mathcal{E}_{a}$ is a regular Dirichlet form. The corresponding generator $L_{a}$ is essentially self -adjoint on $\mathcal{H}_{a}$ (and other natural domains, like $\mathcal{S}(\mathbb{R})$ and $C_{0}^{\infty}(\mathbb{R})$ in $\left.L^{2}\left(\mu_{a}\right)\right)$.

Let $T_{t}^{a}$ be the semigroup associated to $\mathcal{E}_{a}$. It is well known that

$$
T_{t}^{a}: x^{n}:_{a}=e^{-a n t}: x^{n}:_{a} .
$$

$T_{t}^{a}$ is called the Ornstein -Uhlenbeck semigroup (given by $\mu_{a}$ ).

Let $\left(J_{n}^{a}\right)_{n \in \mathbb{N}}$ be the projections of $L^{2}\left(\mu_{a}\right)$ onto $\mathcal{H}_{a}^{n}$. The following results are well known (see e.g. [30]):

Theorem 4.36 The Ornstein -Uhlenbeck semigroup $\left(T_{t}^{a}\right)_{t \geq 0}$ (resp. generator $L_{a}$, Dirichlet form $\mathcal{E}_{a}$ ) admits the following decomposition on $L^{2}\left(\mu_{a}\right)$

$$
\begin{gathered}
T_{t}^{a} u(\omega)=\sum_{n=0}^{\infty} e^{-n a t} J_{n}^{a}(u)(\omega), \quad \omega \in E, u \in L^{2}\left(\mu_{a}\right) \\
\operatorname{Dom}\left(L_{a}\right)=\left\{u \in L^{2}\left(\mathbb{R}, \mu_{a}\right): \sum_{n=0}^{\infty} n^{2}\left\|J_{n}^{a}(u)\right\|^{2}<\infty\right\} \\
L_{a} u=-\sum_{n=1}^{\infty} n a J_{n}^{a}(u) \\
\operatorname{Dom}\left(\mathcal{E}_{a}\right)=\operatorname{Dom}\left(\sqrt{-L_{a}}\right)=\left\{u \in L^{2}\left(\mathbb{R}, \mu_{a}\right): \sum_{n=0}^{\infty} n\left\|J_{n}^{a}(u)\right\|^{2}<\infty\right\} \\
\mathcal{E}_{a}(u, v)=\sum_{n=1}^{\infty} n J_{n}^{a}(u) J_{n}^{a}(v) \quad \forall u, v \in \operatorname{Dom}\left(\sqrt{-L_{a}}\right)
\end{gathered}
$$

where all the sums are converging in the norm $\|\cdot\|_{L^{2}\left(\mu_{a}\right)}$. 
From Theorem 4.31, Remark 4.32 it follows that there is a unique Hunt process

$$
\mathbf{M}_{a}=\left(\Omega, \mathcal{F}_{\infty},\left(X_{t}\right)_{t \geq 0},\left(P_{x}\right)_{x \in \mathbb{R}}\right),
$$

which solves the martingale problem for $\left(L_{a}, \mathcal{H}_{a}\right)$. From the decomposition theorem applied to $u(x)=x$ and further results in [4], where the theory of Dirichlet forms is applied, it follows in particular that there is an $\mathcal{E}_{a}$-exceptional set $N$, such that $\forall x \in E \backslash N, \omega \in \Omega$.

$$
\begin{gathered}
X_{t}(\omega)-x=-a \int_{0}^{t} X_{s}(\omega) d s+B_{t}(\omega) \\
X_{0}(\omega)=x
\end{gathered}
$$

With the notations $\stackrel{\circ}{\mathcal{M}}, \mathcal{N}^{c}$ of Section 2.8 , we have that $\left(B_{t}\right)_{t \geq 0} \in \mathcal{M}$ is a Brownian motion adapted to $\left(\mathcal{F}_{t}\right)_{t \geq 0}$ with zero mean and variance $2 t$ (i.e $P_{x}\left(B_{t} \in A\right)=\int_{A} \frac{1}{\sqrt{2 t}} e^{\frac{-y^{2}}{2 t}} d y$ and $B_{t}(\omega) \in C\left(\mathbb{R}_{+} \in \mathbb{R}\right)$ for every $\omega \in \Omega$ ). Moreover

$$
-a \int_{0}^{t} X_{s}(\omega) d s=\int_{0}^{t} L_{a} X_{s}(\omega) d s \in \mathcal{N}^{c}
$$

From Theorem 4.31 it follows that $\left(X_{t}\right)_{t \geq 0}$ is the unique solution of the initial value problem (90), (91) for every $x \in E \backslash N$. It is well known that $X_{t}$ has a version with continuous paths (as seen, e.g., by Kolmogorov -Prohorov criterium), that can be started at every point $x \in \mathbb{R}$. See e.g. [10], [43].

Of course, in this case, stronger results (uniqueness of strong solutions of $(90),(91)$ on the Wiener space) can be obtained by using more "classical" methods (see e.g. [10],[43]), rather than the theory of Dirichlet forms, but our aim was just to provide a simple illustration of our methods.

Remark 4.37 In [8] several results have been established concerning the subordinates of the Ornstein-Uhlenbeck semigroups and process. Let us recall them briefly. Let $\left(T_{t}^{a, f}\right)_{t \geq 0}$ be the subordinate semigroup of the Ornstein -Uhlenbeck semigroup $\left(T_{t}^{a}\right)_{t \geq 0}$ w.r.t. the Bernstein function $f$ on $L^{2}\left(\mu_{a}\right)$. Let $L_{a}^{f}$ (resp. $\mathcal{E}_{a}^{f}$ ) be the corresponding generator (resp. Dirichlet form).

The following facts have been established:

$$
\begin{gathered}
\text { i) } T_{t}^{a, f} F=\sum_{n=0}^{\infty} e^{-f(n a) t} J_{n}^{a}(F), \quad F \in L^{2}\left(\mu_{a}\right) \\
\text { ii) } \operatorname{Dom}\left(L_{a}^{f}\right)=\left\{u \in L^{2}\left(\mu_{a}\right): \sum_{n=0}^{\infty} f(n a)^{2}\left\|J_{n}^{a}(u)\right\|^{2}<\infty\right\} \\
L_{a}^{f} u=-\sum_{n=1}^{\infty} f(n a) J_{n}^{a}(u) \\
i i i) \operatorname{Dom}\left(\mathcal{E}_{a}^{f}\right)=\operatorname{Dom}\left(\sqrt{-L_{a}}\right)=\left\{u \in L^{2}\left(\mu_{a}\right): \sum_{n=0}^{\infty} f(n a)\left\|J_{n}^{a}(u)\right\|^{2}<\infty\right\} \\
\mathcal{E}_{a}^{f}(u, v)=\sum_{n=1}^{\infty} f(n a) J_{n}^{a}(u) J_{n}^{a}(v) \quad \forall u, v \in \operatorname{Dom}\left(\sqrt{-L_{a}^{f}}\right) \\
i v) \quad T_{t}^{a, f}(\exp (i \xi x))=e^{-a^{-1} \xi^{2} / 2} \sum_{n=1}^{\infty} \frac{(i \xi)^{n} a^{-n / 2}}{\sqrt{n !}} e^{-t f(n a)}: x^{n}:_{a}
\end{gathered}
$$


where all the sums are converging in the $\|\cdot\|_{L^{2}\left(\mu_{a}\right)}$-norm.

As a corollary from Theorem 3.8, Theorem 4.16, Theorem 4.35 and Theorem 4.33 one then has that $\mathcal{E}_{a}^{f}$ is a regular Dirichlet form, and it is the closure of $\left(\mathcal{E}_{a}^{f}, \mathcal{H}_{a}\right)$. The generator $L_{a}^{f}$ is essentially self -adjoint on $\mathcal{H}_{a}$.Up to $\mu_{a}$-equivalence there is a unique $\mu_{a}$-symmetric, $\mu_{a}$-tight special standard process $\mathbf{M}_{a}^{f}=\left(\Omega, \mathcal{F}_{\infty},\left(\mathbf{X}_{t}^{f}\right)_{t \geq 0},\left(P_{z}\right)_{z \in X}\right)$ satisfying the MP for $(-f(a), D)$ with $D=\mathcal{H}_{a}$.

In [8] we also proved corresponding results for generalized Ornstein -Uhlenbeck processes on abstract Wiener spaces resp. on the the space of tempered distributions $S^{\prime}\left(\mathbb{R}^{d}\right)$. We also constructed the corresponding stochastic differential equations and proved uniqueness of the pathwise solutions of the corresponding initial valued problems, with the solutions being uniquely defined by the finite dimensional projections.

\section{Dedication}

The second author dedicates this work to the memory of her father Ulrich Rüdiger.

\section{Acknowledgements}

We thank Michael Röckner and Jiang Lun Wu for interesting discussions. The support of Luciano Tubaro and Alberto Valli at the Mathematics Department of the University of Trento is also gratefully acknowledged, as well as financial support by SFB 611.

\section{REFERENCES}

1. S. Albeverio. Theory of Dirichlet forms and applications, pp. 4-105 in S. Albeverio, W. Schachermayer, M. Talagrand, Lectures on Probability Theory and Statistics, Ed. P. Bernard, Proc. St. Flour Lectures 2000, Lect. Notes Maths. 1816, Springer Verlag, Berlin (2003).

2. S. Albeverio, R. Höegh-Krohn. Quasi-invariant measures, symmetric diffusion processes and quantum fields. pp. 11-59 in "Les méthodes mathématiques de la théorie quantique des champs", Colloques Internationaux du Centre Nat. Rech. Sci. Marseille, juin 75, CNRS, 23-27 (1976).

3. S. Albeverio, R. Höegh-Krohn. Dirichlet forms and diffusion processes on rigged Hilbert spaces. Z. Wahr. verw. Geb. 40, 1-57 (1977).

4. S. Albeverio, M. Röckner. Stochastic differential equations in infinite dimension: solutions via Dirichlet forms. Prob. Probab. Th. Rel. Fiels 89, 347-386 (1992).

5. S. Albeverio, M. Röckner. Classical Dirichlet forms on topological vector spaces - the construction of the associated diffusion process. Probab. Th. Rel. Fields 83, 405-434 (1989).

6. S. Albeverio, M. Röckner. Classical Dirichlet forms on topological vector spaces closability and a Cameron-Martin formula. J. Funct. Anal. 88, 395-436 (1990).

7. S. Albeverio, M. Röckner. Dirichlet form methods for uniqueness of martingale problems and applications. Proceedings of the "1993 Summer Research Institute on Stochastic Analysis, Cornell, July 1993", Eds. R. Durrett et al., Symp. AMS.

8. S. Albeverio, B. Rüdiger. Infinite dimensional Stochastic Differential Equations obtained by subordination and related Dirichlet forms, J. Funct. Anal. 204, 1, 122-156 (2003). 
9. S. Albeverio, B. Rüdiger, J.-L. Wu. Analytic and Probabilistic Aspects of Lévy Processes and Fields in Quantum Theory. Pp. 187-224 in Lévy Processes:Theory and Applications, eds. O.Barndorff -Nielsen, T. Mikosch, S. I. Resnick, Birkhäuser Verlag, Basel (2001).

10. L. Arnold. Stochastic Differential Equations: Theory and Applications. J. Wiley, New York (1974).

11. S. Albeverio, S. Song. Closability and resolvent of Dirichlet forms perturbed by jumps. Pot. Anal. 2, $115-130$ (1993).

12. H. Bauer. Wahrscheinlichkeitstheorie und Grundzüge der Masstheorie. De Gruyter Lehrbuch. 2 Auflage. Walter de Gruyter. Berlin - New York (1974).

13. Ch. Berg, Kh. Boyadzhiev, R. de Laubenfels. Generation of generators of holomorphic semigroups. J. Austral. Math. Soc. (Ser A) 55, 246-269 (1993).

14. J. Bertoin. Lévy processes. Cambridge Tracts in Mathematics 121, Cambridge University Press (1996).

15. R.M. Blumental, R.K. Getoor. Markov Processes and Potential Theory. Mathematics 29, Academic Press, New York, London (1968).

16. V. Bogachev, P. Lescot, M. Röckner. The martingale problem for pseudo differential operators on infinite dimensional spaces, Nagoya Math. J. 153, 101-118 (1999).

17. S. Bochner, Harmonic Analysis and the Theory of Probability. California Monographs in Math. Sci., University of California Press, Berkeley, CA (1955).

18. N. Bouleau. Operateur carré du champ, subordinateurs et processus de Dirichlet, Seminar on potential theory, Paris, No.7, 134-156, Lecture Notes in Math., 1061, Springer Verlag, Berlin (1984).

19. N. Bouleau and F. Hirsch. Dirichlet Forms and Analysis on Wiener Space. Studies in Mathematics 14, Walter de Gruyter, Berlin (1991).

20. N. Bouleau and F. Hirsch, Générateurs étendus et subordination au sens de Bochner. C. R. Acad. Sci. Paris Sér. I Math. 296, 16, 709-712 (1983).

21. M. F. Chen. From Markov Chains to Non-Equilibrium Particle Systems. World Scientific, Singapore (1992).

22. B. Debrulle, F. Gramer, D. Sornette, eds. Scale Invariance and Beyond. Springer Verlag, Berlin (1997).

23. C. Dellacherie, P. A. Meyer. Probabilities and Potentials B. Theory of Martingales. Mathematics Studies 72, North-Holland Publishing Co, Amsterdam, New York (1982).

24. A. Eberle. Uniqueness and non -uniqueness of semigroups generated by singular diffusion operators. LN Maths. 1718, Springer Verlag, Berlin (1999).

25. W. E. Feller. An introduction to probability theory and its applications. 2nd edn, vol.2. Wiley, New York (1971).

26. M. Fukushima. Dirichlet Forms and Markov Processes. North Holland, Amsterdam (1980).

27. M. Fukushima, Y. Oshima, M. Takeda. Dirichlet Forms and Symmetric Markov Processes. Walter de Gruyter, Berlin (1994). 
28. F. Hirsch. Générateurs étendus et subordination au sens de Bochner. Seminar on potential theory, Paris, No.7, 134-156, Lecture Notes in Math., 1061, Springer Verlag, Berlin (1984).

29. F. Hirsch, S. Song. Inequalities for Bochner's subordinators of two -parameter symmetric Markov processes. Ann. Inst. H. Poincaré Probab. Statist. 32, 5, 589600, (1996).

30. T. Hida, H.-H. Kuo, J. Potthoff, L. Streit. White Noise: An Infinite Dimensional Calculus. Kluwer Academic, Dordrecht (1993).

31. N. Jacob. Pseudo-Differential Operators and Markov Processes. Akademie Verlag, Berlin (1996).

32. N. Jacob. Pseudo-Differential Operators and Markov Processes: 1. Fourier Analysis and Semigroups. World Scientific, Singapore (2001).

33. N. Jacob. Further pseudodifferential operators generating Feller semigroups and Dirichlet forms. Revista Matemática Iberoamericana 9, (1993).

34. G. Jona-Lasinio, P. K. Mitter. On the stochastic quantization of field theory. Comm. Math. Phys. 101, 409-436 (1985).

35. T. Kato. Perturbation Theory for Linear Operators. Grundlehren der mathematischen Wissenschaften 132 Springer-Verlag, Berlin, Heidelberg, New York (1980).

36. J. U. Löbus. Closability of positive symmetric bilinear forms under non-regularity assumptions and its probabilistic counterpart. Preprint (1996).

37. Z.-M. Ma, M. Röckner. An Introduction on the Theory of (Non-Symmetric) Dirichlet Forms. Springer-Verlag, Berlin (1992).

38. Mc Gillivray. A recurrence condition for some subordinated strongly local Dirichlet form, Forum Math. 9, 229-246 (1997).

39. R. Mikulievikius and B.L. Rozovskii. Martingale problems for stochastic PDEs. pp. 251-333 in "Stochastic Partial Differential Equations. Six Perspectives" (eds. R. Carmona, B.L. Rozovskii). AMS, Rhode Island (1999).

40. D. Nualart. The Malliavin Calculus and Related Topics. Probability and its Applications. Springer-Verlag, Berlin (1995).

41. H. Ôkura. Recurrence and transience criteria for subordinated symmetric Markov processes, Forum Math. 14, 121-146 (2002).

42. R. S. Philipps. On the generation of semigroups of linear operators. Pacific J. Math. 2, 343-369 (1952).

43. D. Revuz, M. Yor. Continuous Martingales and Brownian Motion. Grundlehren der mathematischen Wissenschaften 293. A Series of Comprehensive Studies in Mathematics, Berlin, Heidelberg, Springer Verlag (1991).

44. B. Rüdiger, J.-L. Wu. Construction by subordination of processes with jumps on infinite dimensional state spaces and corresponding non local Dirichlet forms. Pp. 559-571 in Stochastic Processes, Physics and Geometry: New Interplays. A Volume in Honor of Sergio Albeverio. Eds. F. Gesztesy et al., Proceedings of the International Conference on Infinite Dimensional (Stochastic) Analysis and Quantum Physics (Leipzig 1999), the Canadian Mathematical Society Conference Proceedings Series 29(2000). 
45. K. I. Sato. Lévy Processes and Infinitely Divisible Distributions. Cambridge studies in advanced mathematics 68. Cambridge University Press (1999).

46. R. Schilling. Subordination in the sense of Bochner and related functional calculus, J. Austr. Math, Soc. Ser. A 64, 368-396 (1998).

47. M. F. Shlesinger, G. M. Zavslavsky and U. Frisch, eds. Lévy Flights and Related Topics in Physics. Springer-Verlag (1995).

48. B. Simon. The $P(\varphi)_{2}$ Euclidean (Quantum) Field Theory. Princeton Univ. Press (1975).

49. B. Schmuland. Dirichlet forms: some infinite -dimensional examples. Canad. J. Statist. 27 4, 683-700 (1999).

50. B. West. An Essay of the Importance of Being Non Linear. Lecture Notes in Biomathematics 62, Springer-Verlag, Berlin (1985).

51. K. Yosida. Fractional powers of infinitesimal generators and the analyticity of the semigroup generated by them. Proc. Japan. Acad. 36, 86-89 (1960). 